\title{
Character States and Generator Matrix Elements for
}

$$
S p(4) \supset S U(2) \times U(1)
$$

\author{
N. Hambli, J. Michelson and R. T. Sharp \\ Department of Physics, McGill University \\ 3600 University St., Montréal, Québec H3A-2T8 Canada f
}

March 19, 2018

${ }^{*}$ Research supported in part by the Natural Sciences and Engineering Research Council of Canada and by the Fonds FCAR du Québec. 


\begin{abstract}
A new set of polynomial states (to be called character states) are derived for $S p(4)$ reduced to its $S U(2) \times U(1)$ subgroup, and the relevant generator matrix elements are evaluated. The group-subgroup in question is that of the seniority model of nuclear physics.
\end{abstract}




\section{Introduction}

The group-subgroup $S p(4) \supset S U(2) \times U(1)$ finds application in the nuclear seniority model (Flowers 1952). Polynomial basis states (in the states of the $S p(4)$ fundamental representations) have been given by Hecht (1965), Parikh (1965), Ahmed and Sharp (1970) and by Smirnov and Tolstoy (1973). In this paper we present new polynomial basis states, which we christen "character states"; we believe then to be simpler than any of the earlier ones. Analogous states have been given recently for $S U(3)$ and $S O(5)$ reduced according to their finite Demazure-Tits subgroup (de Guise and Sharp 1991), for $S O(7)$ reduced according to $S U(2)^{3}$ (Burdik, Cummins, Gaskell and Sharp 1992) and for $G_{2}$ reduced according to $S U(3)$ (Farell, Lam and Sharp 1993).

In Section (2) we discuss character states in the context of $S p(4) \supset S U(2) \times U(1)$. Section (3) deals with generator matrix elements for the degenerate representations $(a, 0)$ and $(0, b)$. Section (5) contains some concluding remarks.

\section{Character States For $S p(4) \supset S U(2) \times U(1)$}

The basis states of the $S p(4)$ representation $(a, b)$ are polynomials of degrees $a, b$ in the states of the respective fundamental representations. Hence only stretched IR's (representation labels additive) in the direct product of $a$ copies of $(1,0)$ and $b$ copies of $(0,1)$ are to be retained. 
Consider the quadratic direct products

$$
\begin{aligned}
(1,0)^{2}{ }_{10} & =(2,0)_{10}, \\
(0,1)^{2}{ }_{15} & =(0,2)_{14}+(0,0)_{1}, \\
(1,0) \times(0,1)_{20} & =(1,1)_{16}+(1,0)_{4} .
\end{aligned}
$$

The square of a representation above means the symmetric (polynomial) part of the direct product of two copies. A subscript on a representation or product is its dimension. The stretched, or wanted, part of each product is the first representation on the right; the states of the other representations are unwanted for the purpose of forming our polynomial basis.

The $S p(4)$ character generator may be written

$$
X(A, B ; M, Z)=\frac{1}{\beta \eta \zeta}\left[\frac{1}{\xi \alpha \theta}+\frac{\gamma}{\alpha \theta \gamma}+\frac{\kappa}{\theta \gamma \kappa}+\frac{\delta}{\gamma \kappa \delta}+\frac{\alpha \delta}{\gamma \delta \alpha}\right]
$$

we have adopted the space-saving convention that a variable in a denominator stands for unity minus that variable. To stress our interpretation of the character generator as generating function for basis states we have expressed $X$ in terms of the fundamental representation states (see figure 1). In the power series expansion of (2.2) each term of degree $a$ in the $(1,0)$ variables and degree $b$ in the $(0,1)$ variables represents one state of the representation $(a, b)$. The states thus defined are complete and non redundant. We call them character states. We remark that they are contaminated by unwanted states belonging to lower representations; that does not matter for the purpose of computing generator matrix elements. For $X$ as a generating function for 
characters, or weights, the following substitutions should be made:

$$
\begin{aligned}
& \alpha \rightarrow A M^{\frac{1}{2}} Z^{\frac{1}{2}}, \quad \beta \rightarrow A M^{\frac{1}{2}} Z^{-\frac{1}{2}}, \quad \gamma \rightarrow A M^{-\frac{1}{2}} Z^{\frac{1}{2}}, \quad \delta \rightarrow A M^{-\frac{1}{2}} Z^{-\frac{1}{2}} \\
& \eta \rightarrow B Z, \quad \xi \rightarrow B M, \quad \zeta \rightarrow B Z^{-1}, \quad \kappa \rightarrow B M^{-1}, \quad \theta \rightarrow B .
\end{aligned}
$$

The variables $A$ and $B$ are dummies which carry the $S p(4)$ representation labels $a$ and $b$ as exponents; $M$ carries the $S U(2)$ weight $m$ and $Z$ the $U(1)$ label $z$. Then in the expansion of $X$,

$$
X(A, B ; M, Z)=\sum_{a b m z} A^{a} B^{b} M^{m} Z^{z} C_{a b m z}
$$

the coefficient $C_{a b m z}$ is the multiplicity of the weight $(m, z)$ in the $\operatorname{IR}(a, b)$. The character generator (2.2) with the substitutions (2.3) agrees with earlier versions (Gaskell, Peccia and Sharp 1978, Patera and Sharp 1979, de Guise and sharp 1991) when the weights are expressed in the same basis and the terms put over a common denominator.

Examination of (2.2) reveals that certain pairs of variables, namely $\alpha \kappa, \gamma \xi, \delta \xi, \delta \theta$, $\xi \kappa$ never appear in the same term and therefore these products never appear in the expression for a state; we say they are incompatible. Each incompatible pair appears as one term in the expression for one of the unwanted states on the right hand side of (2.1). Setting the unwanted states equal to zero and solving for the incompatible pairs gives the following substitutions by which incompatible pairs may be eliminated in favour of compatible ones when they arise in the course of a calculation:

$$
\alpha \kappa=\delta \eta+\frac{\sqrt{2}}{2} \gamma \theta
$$




$$
\begin{aligned}
\gamma \xi & =-\beta \eta+\frac{\sqrt{2}}{2} \alpha \theta, \\
\delta \xi & =\alpha \zeta+\frac{\sqrt{2}}{2} \beta \theta, \\
\delta \theta & =\sqrt{2} \beta \kappa+\sqrt{2} \gamma \zeta, \\
\xi \kappa & =\eta \zeta+\frac{1}{2} \theta^{2} .
\end{aligned}
$$

It is shown by Farell, Lam and Sharp 1994, and Hambli and Sharp 1995 that elementary unwanted states are all of degree 2 and hence that elementary incompatibilities are between pairs of states only, a fact verified straightforwardly for $S p(4)$.

We complete this section by giving the $S p(4) \supset S U(2) \times U(1)$ branching rules generating function (Sharp and Lam 1969 give the equivalent integrity basis):

$$
\frac{1}{\left(1-A T^{\frac{1}{2}} Z^{\frac{1}{2}}\right)\left(1-A T^{\frac{1}{2}} Z^{-\frac{1}{2}}\right)(1-B Z)\left(1-B Z^{-1}\right)}\left[\frac{1}{(1-B T)}+\frac{A^{2}}{\left(1-A^{2}\right)}\right]
$$

The elements of (2.6) may be interpreted as the highest states of subgroups IR's contained in low $S p(4)$ IR's:

$$
\begin{aligned}
& \alpha \sim A T^{\frac{1}{2}} Z^{\frac{1}{2}}, \quad \beta \sim A T^{\frac{1}{2}} Z^{-\frac{1}{2}}, \quad \alpha \delta-\beta \gamma \sim A^{2}, \\
& \eta \sim B Z, \quad \zeta \sim B Z^{-1}, \quad \xi \sim B T .
\end{aligned}
$$

Thus the highest state of a subgroup IR may be written, in unnormalized form, and representation labels suppressed,

$$
|t, t, z ; v\rangle=\alpha^{x} \beta^{y} \eta^{u} \zeta^{v} \xi^{w}(\alpha \delta-\beta \gamma)^{s}
$$


with

$$
\begin{aligned}
& a=x+y+2 s, \quad b=u+v+w \\
& t=\frac{1}{2}(x+y)+w, \quad z=\frac{1}{2}(x-y)+u-v,
\end{aligned}
$$

and $s w=0$. We allow $v$ to play the role of the missing label.

We may distinguish two types of state, according to whether (type I) $s=0$ (and $t \geq a / 2$ ) or (type II) $w=0$ (and $t \leq a / 2$ ). Solving (2.9) for $x, y, u$ and $w$ or $s, 2.8$ ) becomes

$$
|t, t, z ; v\rangle=\alpha^{t+z-b+2 v} \beta^{a+b-t-z-2 v} \eta^{\frac{a}{2}+b-t-v} \zeta^{v} \xi^{t-\frac{a}{2}}
$$

for type I, and, for type II

$$
|t, t, z ; v\rangle=\alpha^{t+z-b+2 v} \beta^{b+t-z-2 v}(\alpha \delta-\beta \gamma)^{\frac{a}{2}-t} \eta^{b-v} \zeta^{v}
$$

For $t=a / 2$ the two types of state coincide.

The $S U(2)$ root generators are

$$
\begin{aligned}
& T_{+}=\alpha \partial_{\gamma}+\beta \partial_{\delta}+\sqrt{2}\left(\xi \partial_{\theta}+\theta \partial_{\kappa}\right), \\
& T_{-}=\gamma \partial_{\alpha}+\delta \partial_{\beta}+\sqrt{2}\left(\theta \partial_{\xi}+\kappa \partial_{\theta}\right) .
\end{aligned}
$$

The other $6 S p(4)$ root generators constitute an $S U(2)$ vector $G$ with $z=1$,

$$
G_{+1}=\alpha \partial_{\delta}+\eta \partial_{\kappa}+\xi \partial_{\zeta}
$$




$$
\begin{aligned}
G_{-1} & =-\gamma \partial_{\beta}+\kappa \partial_{\zeta}+\eta \partial_{\xi} \\
G_{0} & =\frac{\sqrt{2}}{2}\left(\gamma \partial_{\delta}-\alpha \partial_{\beta}\right)+\theta \partial_{\zeta}-\eta \partial_{\theta}
\end{aligned}
$$

and a vector $\bar{G}$ with $z=-1$,

$$
\begin{aligned}
\overline{G_{+1}} & =\beta \partial_{\gamma}-\xi \partial_{\eta}-\zeta \partial_{\kappa}, \\
\overline{G_{-1}} & =-\delta \partial_{\alpha}-\kappa \partial_{\eta}-\zeta \partial_{\xi}, \\
\overline{G_{0}} & =\frac{\sqrt{2}}{2}\left(\delta \partial_{\gamma}-\beta \partial_{\alpha}\right)+\zeta \partial_{\theta}-\theta \partial_{\eta} .
\end{aligned}
$$

$G$ and $\bar{G}$ are hermitian conjugate with

$$
\overline{G_{i}}=(-1)^{i} G_{-i}^{\dagger}
$$

We will save considerable work later by noticing that under the substitutions $\alpha \leftrightarrow$ $\delta, \beta \leftrightarrow \gamma, \eta \leftrightarrow \zeta, \xi \leftrightarrow \kappa$, we have $G_{i} \leftrightarrow-\overline{G_{-i}}$ and that under the same substitutions we have for the type I states (2.10) $|t, m, z ; v\rangle \leftrightarrow|t,-m,-z ; a / 2+b-t-v\rangle$ while for type II states (2.11) $|t, m, z ; v\rangle \leftrightarrow|t,-m,-z ; b-v\rangle$.

\section{Generator Matrix Elements For Degenerate Representa- tions}

For the degenerate IR's $(a, 0)$ and $(0, b)$ there is no missing label and the basis states are orthogonal and hence can be normalized straightforwardly. Since in that sense they can be handled more satisfactorily than generic states $(a>0, b>0)$ we treat them separately. 
For $(a, 0)$, according to (2.11) with $v=0$, the highest state of an $S U(2)$ IR is (we suppress the label $a$ )

$$
|t, t, z\rangle=N_{t} z \alpha^{t+z} \beta^{t-z}(\alpha \delta-\beta \gamma)^{\frac{a}{2}-t}
$$

The branching rule is $a / 2 \geq t \geq|z|$ with $2 t$ and $2 z$ having the parity of $a$.

$(a, 0)$ states are not contaminated by unwanted states and can be normalized by standard methods. For brevity write

$$
|h\rangle=N_{h} \alpha^{f} \beta^{g}(\alpha \delta-\beta \gamma)^{h},
$$

and equate

$$
\langle h+1|\alpha \delta-\beta \gamma| h\rangle=\left\langle h\left|\partial_{\alpha} \partial_{\delta}-\partial_{\beta} \partial_{\gamma}\right| h+1\right\rangle
$$

to obtain a recursion relation for $N_{h}$, whose solution is, for $N_{t} z$,

$$
N_{t z}=\sqrt{\frac{(2 t+1) !}{(t+z) !(t-z) !\left(\frac{a}{2}-t\right) !\left(\frac{a}{2}+t+1\right) !}}
$$

We can write

$$
G_{0}|t, t, z\rangle=A|t+1, t, z+1\rangle+B|t, t, z+1\rangle
$$

where the matrix elements $A$ and $B$ will now be determined. Apply $T_{+}$to (3.5) with the result

$$
\sqrt{2} G_{1}|t, t, z\rangle=\sqrt{2(t+1)} A|t+1, t+1, z+1\rangle
$$

from which

$$
\begin{aligned}
A & =\left\langle t+1, t, z+1\left|G_{0}\right| t, t, z\right\rangle=\frac{\frac{a}{2}-t}{\sqrt{t+1}} \frac{N_{t z}}{N_{t+1} z+1} \\
& =\frac{1}{t+1} \sqrt{\frac{(t+z+1)(t+z+2)\left(\frac{a}{2}-t\right)\left(\frac{a}{2}+t+2\right)}{2(2 t+3)}} .
\end{aligned}
$$


Inserting this value for $A$ in (3.5) we obtain for $B$

$$
\begin{aligned}
B & =\left\langle t, t, z+1\left|G_{0}\right| t, t, z\right\rangle=-\frac{\left(\frac{a}{2}+1\right)(t-z)}{\sqrt{2}(t+1)} \frac{N_{t z}}{N_{t z+1}}, \\
& =-\frac{\frac{a}{2}+1}{\sqrt{2}(t+1)} \sqrt{(t+z+1)(t-z)} .
\end{aligned}
$$

To obtain a matrix element of $G_{0}$ in which $G_{0}$ reduces $t$ by unity make the following transformations:

$$
\begin{aligned}
\left\langle t+1, t, z+1\left|G_{0}\right| t, t, z\right\rangle & =-\left\langle t+1,-t,-z-1\left|\overline{G_{0}}\right| t,-t,-z\right\rangle, \\
& =-\left\langle t,-t,-z\left|G_{0}\right| t+1,-t,-z-1\right\rangle .
\end{aligned}
$$

The first step follows from the substitutions in the paragraph following (2.15) and the second is a consequence of (2.15).

For the reduced matrix element of $G$ we get

$$
\begin{aligned}
\langle t+1, z+1\|G\| t, z\rangle & =\sqrt{\frac{(t+z+1)(t+z+2)\left(\frac{a}{2}-t\right)\left(\frac{a}{2}+t+2\right)}{2(t+1)}}, \\
\langle t, z+1\|G\| t, z\rangle & =-\left(\frac{a}{2}+1\right) \sqrt{\frac{(2 t+1)(t+z+1)(t-z)}{2 t(t+1)}}, \\
\langle t-1, z+1\|G\| t, z\rangle & =\sqrt{\frac{(t-z-1)(t-z)\left(\frac{a}{2}-t+1\right)\left(\frac{a}{2}+t+1\right)}{2 t}} .
\end{aligned}
$$

Because of $(2.15)$ the reduced matrix elements of $\bar{G}$ are given in terms of those of $G$ by the relation

$$
\langle t+k, z-1\|\bar{G}\| t, z\rangle=-(-1)^{k}\langle t, z\|G\| t+k, z-1\rangle .
$$

We turn to $(0, b)$ states. According to (2.10) with $v=(b-t-z) / 2$ the highest state of a subgroup multiplet is

$$
|t, t, z\rangle=N_{t z} \eta^{\frac{(b-t+z)}{2}} \zeta^{\frac{(b-t-z)}{2}} \xi^{t}
$$


The branching rule is $b-|z| \geq t \geq 0$. The $S U(2)$ ladder generators are now

$$
\begin{aligned}
& T_{+}=\sqrt{2}\left(\xi \partial_{\theta}+\theta \partial_{\kappa}\right), \\
& T_{-}=\sqrt{2}\left(\theta \partial_{\xi}+\kappa \partial_{\theta}\right) .
\end{aligned}
$$

The components of $G$ and $\bar{G}$ are

$$
\begin{gathered}
G_{+1}=\eta \partial_{\kappa}+\xi \partial_{\zeta}, \\
G_{-1}=\kappa \partial_{\zeta}+\eta \partial_{\xi}, \\
G_{0}=\theta \partial_{\zeta}-\eta \partial_{\theta}, \\
\overline{G_{+1}}=-\xi \partial_{\eta}-\zeta \partial_{\kappa}, \\
\overline{G_{-1}}=-\kappa \partial_{\eta}-\zeta \partial_{\xi}, \\
\overline{G_{0}}=\zeta \partial_{\theta}-\theta \partial_{\eta} .
\end{gathered}
$$

Again $\overline{G_{i}}=(-1)^{i} G_{-i}^{\dagger}$. The basis states are contaminated by states containing as a factor the unwanted scalar $\eta \zeta-\xi \kappa+\theta^{2} / 2$.

Applying $\overline{G_{-1}}$ to $|t, t, z\rangle$ yields

$$
\overline{G_{-1}}|t, t, z\rangle=A|t+1, t-1, z-1\rangle+C|t-1, t-1, z-1\rangle .
$$

( $t$ changes only by \pm 1 because it has the parity of $b+z$.) We must now find the matrix elements $A$ and $C$. Application of $T_{+}^{2}$ to (3.16) gives

$$
\overline{G_{1}}|t, t, z\rangle=A \sqrt{(t+1)(2 t+1)}|t+1, t+1, z-1\rangle,
$$


from which

$$
\begin{aligned}
A & =\left\langle t+1, t-1, z-1\left|\overline{G_{-1}}\right| t, t, z\right\rangle \\
& =-\frac{1}{2} \frac{(b-t+z)}{\sqrt{(t+1)(2 t+1)}} \frac{N_{t z}}{N_{t+1} z-1}
\end{aligned}
$$

Then (3.16) yields immediately

$$
\begin{aligned}
C & =\left\langle t-1, t-1, z-1\left|\overline{G_{-1}}\right| t, t, z\right\rangle \\
& =-\frac{t(b+t+z+1)}{2 t+1} \frac{N_{t z}}{N_{t-1 z-1}}
\end{aligned}
$$

Applying $G_{1}$ to $|t-1, t-1, z-1\rangle$ gives

$$
\left\langle t, t, z\left|G_{1}\right| t-1, t-1, z-1\right\rangle=\frac{1}{2}(b-t-z+2) \frac{N_{t-1 z-1}}{N_{t z}} .
$$

Because $\overline{G_{-1}}=-G_{1}^{\dagger}$ we can equate the right hand side of $(3.20)$ to the negative of the right hand side of (3.19), getting a recursion relation for the normalization constant

$$
\frac{N_{t z}}{N_{t-1} z-1}=\sqrt{\frac{(b-t-z+2)(2 t+1)}{2(b+t+z+1) t}}
$$

whose solution is

$$
N_{t z}=\phi \sqrt{\frac{(2 t+1) ! !}{2^{\frac{1}{2}(b+t-z)} t !\left(\frac{b-t-z}{2}\right) !(b+t+z+1) ! !}}
$$

where $\phi$ is constant when $t$ and $z$ are given the same increment. Increasing $t$ and $z$ each by $(b-t-z) / 2$ takes us to the state

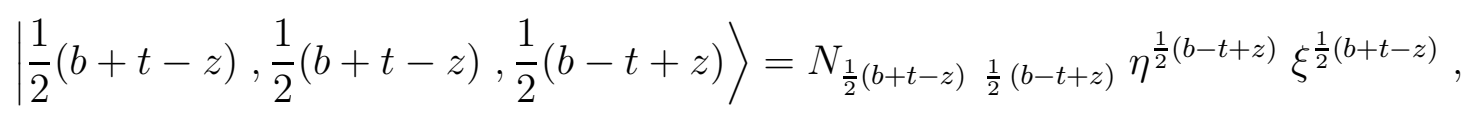


which is on the boundary of the $(0, b)$ weight diagrams and uncontaminated. It is easily normalized:

$$
N_{\frac{1}{2}(b+t-z) \frac{1}{2}(b-t+z)}=\frac{1}{\sqrt{\left(\frac{b-t+z}{2}\right) !\left(\frac{b+t-z}{2}\right) !}} .
$$

Using (3.24) in (3.22) determines $\phi$ to be

$$
\phi=\sqrt{\frac{2^{\frac{1}{2}(b+t-z)}(2 b+1) ! !}{\left(\frac{b-t+z}{2}\right) !(b+t-z+1) ! !}} .
$$

(3.22) then gives for $N_{t} z$

$$
N_{t z}=\sqrt{\frac{(2 t+1) ! !(2 b+1) ! !}{t !\left(\frac{b-t-z}{2}\right) !\left(\frac{b-t+z}{2}\right) !(b+t+z+1) ! !(b+t-z+1) ! !}} .
$$

We have normalized the wanted part of the state (3.12) without ever isolating it. As a check consider (3.12) with $b=4, t=0, z=0$, i.e., $N_{00} \eta^{2} \zeta^{2}$. The wanted part of $\eta^{2} \zeta^{2}$, i.e., the part orthogonal to $\eta \zeta\left(\eta \zeta-\xi \kappa+1 / 2 \theta^{2}\right)$ and to $\left(\eta \zeta-\xi \kappa+1 / 2 \theta^{2}\right)^{2}$ is $1 / 63\left(15 \eta^{2} \zeta^{2}+8 \xi^{2} \kappa^{2}+2 \theta^{4}+40 \eta \zeta \xi \kappa-20 \eta \zeta \theta^{2}-8 \xi \kappa \theta^{2}\right)$ whose norm is $20 / 21$ which checks with 3 ) by which $N_{00}=\sqrt{21 / 20}$.

Inserting the value of the normalization constant in (3.18) and (3.19) gives the matrix elements explicitly. For the reduced matrix elements of $G$ we find

$$
\begin{aligned}
& \langle t+1, z-1\|\bar{G}\| t, z\rangle=-\sqrt{(t+1)\left(\frac{b-t+z}{2}\right)(b+t-z+3)}, \\
& \langle t-1, z-1\|\bar{G}\| t, z\rangle=-\sqrt{t\left(\frac{b-t-z+2}{2}\right)(b+t+z+1)} .
\end{aligned}
$$




\section{Concluding Remarks}

We have defined the matrix elements of a generator $G$ between states $|h\rangle$ and $|g\rangle$ of a complete non-redundant set as $(g|G| h)=$ coefficient of $|g\rangle$ in $G|h\rangle$. As long as the states are orthonormal this is equivalent to the usual definition $\langle g|G| h\rangle=$

overlap of $|g\rangle$ with $G|h\rangle$; this is the case when the subgroup used to define the states provides a complete set of labels, as for the states we deal with in this paper.

The basis states are defined as the 'wanted' parts of products of powers of mutually compatible sets of basis states of the fundamental representations.

In a forthcoming paper we will treat generic representations $(a, b)$ of $S p(4)$ in an $S U(2) \times U(1)$ basis; there is then a missing label. There is no real need to orthonormalize the states. 


\section{$5 \quad$ References}

Flowers B H 1952 Studies in $j j$-coupling I Classification of Nuclear and Atomic States. Proc. Roy. Soc. (London) A212 248 - 263

Hecht K T 1965 Some Simple $R_{5}$ Wigner Coefficients and Their Applications. Nucl. Phys. $63177-213$

Parikh J C 1965 The Role of Isospin Pair Correlations for Configurations of the Type $(J)^{N}$. Nucl. Phys. $63214-232$

Ahmed K and Sharp R T 1970 O(5) Bases for Nuclear Seniority Model. J. Math. Phys. $111112-1117$

Smirnov Y F and Tolstoy V N 1973 A New Projected Basis in the Theory of Five-Dimensional Quasi-Spin. Rep. Math. Phys. $497-111$

de Guise H and Sharp R T 1991 Polynomial States for $S U(3)$ and $S O(5)$ in a Demazure-Tits basis. J. Phys. A: Math. Gen. $24557-568$

Burdik Č, Cummins C J, Gaskell R W and Sharp R T 1992 Complete Branching Rules Generating Function for $S O(7) \supset S U(2)^{3}$ and Polynomial Basis States. J. Phys. A: Math. Gen. $4835-4846$

Farell L, Lam C S and Sharp R T $1994 G_{2}$ Generator Matrix Elements for Degenerate Representations in an SU(3) Basis: I. J. Phys. A: Math. Gen. $272761-$ 2771 
Hambli N and Sharp R T 1995 Generator Matrix Elements for $G_{2} \supset S U(3)$ : II. Generic Representation. J. Phys. A: Math. Gen. $282581-2588$

Gaskell R, Peccia A and Sharp R T 1978 Generating Functions for Polynomial Irreducible Tensors. J. Math. Phys. $19727-733$

Patera J and Sharp R T 1979 Generating Functions for Characters of Group Representations and Their Applications Lecture Notes in Physics 94175 - 183 


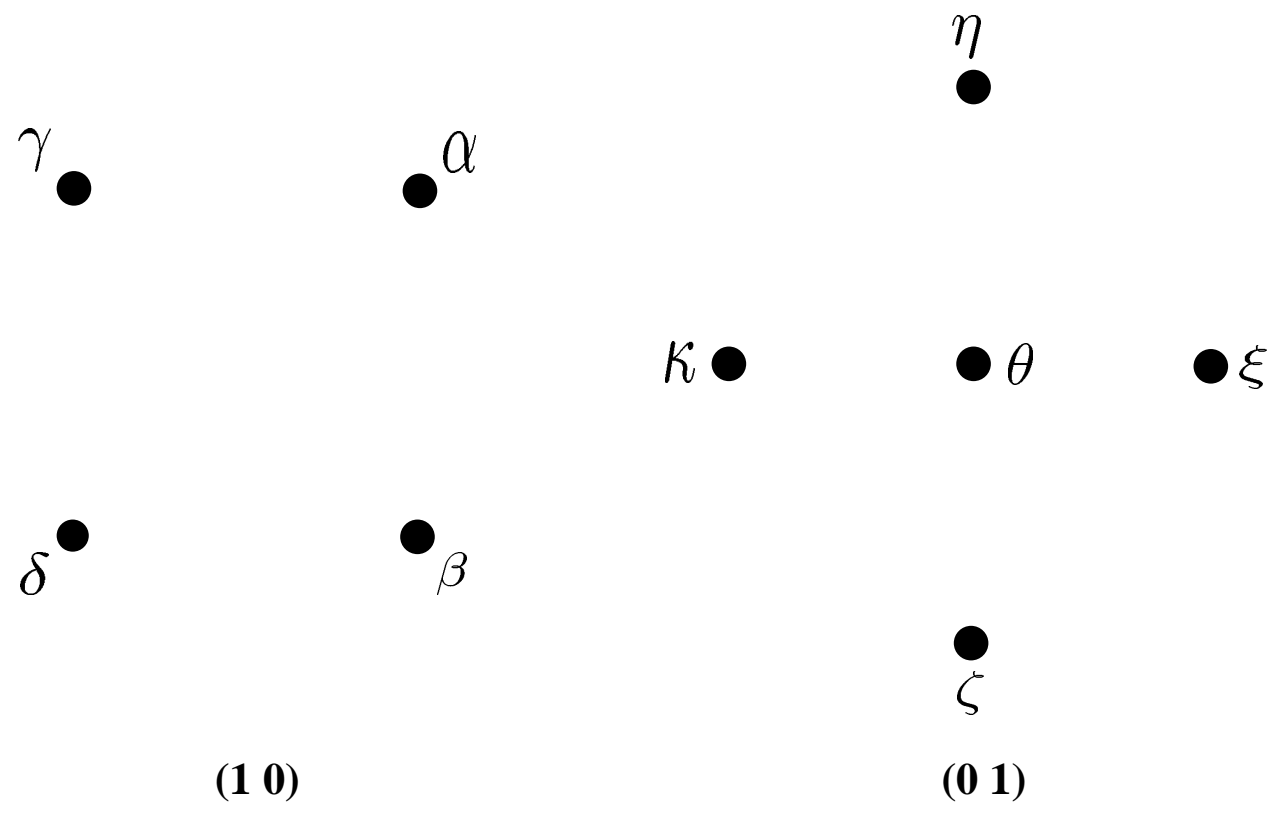

Figure 1: States of the fundamental representations of $S p(4)$. 